\title{
Clinical and misdiagnosed analysis of primary pulmonary lymphoma: a retrospective study
}

\author{
D. Yao', L. Zhang' ${ }^{2}$, P. L. Wu', X. L. Gu², Y. F. Chen', L. X. Wang ${ }^{1}$ and X. Y. Huang ${ }^{1 *}$
}

\begin{abstract}
Background: The primary pulmonary lymphoma (PPL), with a low incidence, was highly misdiagnosed in clinic. The present study analyzes the clinical features, laboratory and imaging data, pathologic characteristics, and summarizes misdiagnosis reasons of PPL cases, aims to provide a better understanding and increase the accuracy of early diagnosis and minimize the misdiagnosis of PPL.
\end{abstract}

Methods: The clinical data of 19 cases were collected from the first affiliated hospital of Wenzhou medical university (PRC) from April 2010 to May 2016. All cases were confirmed by pathology. The process of misdiagnosis was described. This study retrospectively analyzed the incidence, clinical presentation, laboratory examination, Chest CT scan and diagnosis of the cases.

Results: The symptoms of the 19 cases were dyspnea, fever, hemoptysis, chest pain or physical findings without obvious symptoms. Five patients were pneumonia-like, nine patients had lung single nodule or mass and four patients got pleural effusion, which were reported by computed tomography (HRCT) scan. There were 2 cases of Hodgkin lymphoma $(\mathrm{HL})$, and 17 cases of non-Hodgkin lymphoma (NHL). In NHL cases, 12 cases were confirmed mucosa associated lymphoid tissue B lymphoma type, 3 cases were confirmed diffuse large B-cell lymphoma, angioimmunoblastic T-cell lymphoma and ALK positive anaplastic large cell lymphoma were one case separately. Clinical and imaging manifestation of PPL is untypical, but there are still some hints: 1) Fuzzy shadow at the edge of lung mass with air bronchogram; 2) Lung mass shadow stable for a long time; 3) Pneumonia-like changing without infections clinical and lab manifestation. Thirteen patients (68.4\%) were misdiagnosed as pneumonia, lung cancer and tuberculosis initially. The term between initial diagnosis and final diagnosis lasted for half a month up to 2 years, with median time of 6 months. Two cases were misdiagnosed as tuberculosis. One case was misdiagnosed as small cell lung cancer.

Conclusion: Clinical and imaging manifestation of PPL is untypical. Biopsies should be taken actively if the imaging findings don't match the symptoms or the anti-infection treatments to "lung infection" don't work. Accurate diagnosis requires adequate tissue sampling with appropriate ancillary pathologic studies. If clinical manifestation and the diagnosis don't match, repeated biopsy should be ordered.

Keywords: Primary pulmonary lymphoma, Misdiagnosis, Pathology, Biopsy

\footnotetext{
* Correspondence: zjwzhxy@126.com

${ }^{1}$ The First Affiliated Hospital of Wenzhou Medical University \& Key Laboratory

of Heart and Lung, Zhejiang 325035, China

Full list of author information is available at the end of the article
} 


\section{Background}

Primary pulmonary lymphoma (PPL), as an uncommon disease, affects the lung occuring over a broad clinical and pathologic spectrum. PPL, with a low incidence, comprises $<1 \%$ [1] of all non-Hodgkin's lymphoma (NHL), and $0.5 \%$ of all primary pulmonary malignant tumors [2, 3]. Traditionally, it has been defined as a clonal lymphoid proliferation affecting one or both lungs (parenchyma and/or bronchi) in a patient with no clinical, pathological, or radiographic evidence of lymphoma elsewhere, either in the past, or at present, or for 3 months after presentation $[4,5]$. The vast majority are type of low-grade mucosa-associated lymphoid tissue [6-10]. Although there have been several retrospective reports, few studies were investigated in the Asian population [8-10].

PPL usually occurs with nonspecific clinical features, is highly misdiagnosed in clinic. In order to provide a better understanding of this disease, increase the accuracy of early diagnosis and minimize misdiagnosis, this research was to analyze and summarize the misdiagnosis reasons of PPL based on the clinical features, the laboratory and imaging examinations, and the pathologic characteristics of PPL cases from our hospital during April 2010 to May 2016 while referring to relative literature.

\section{Methods}

The clinical data of 19 cases of histologically proven PPL were collected from the first affiliated hospital of Wenzhou Medical University from April 2010 to May 2016. At the same time, there were 760 cases of lymphoma collected in the hospital. All the 19 cases were confirmed by pathology. Medical records about clinical characteristic, the time of diagnosis, treatment and follow-up were systematically reviewed, including clinical features, laboratory findings, radiographs, bronchoscopic features and pathological data. Ethical approval for this investigation was obtained from the Research Ethics Committee, the First Affiliated Hospital of Wenzhou Medical University. And all patients consent to participate and all data were consent for publish.

\section{Results}

\section{Clinical manifestation and laboratory examination}

Within the 19 cases, 11 male and eight female patients were described with a median age of 51.8 years old (range 19 to 71 years). The symptoms (Table 1) of the 19 cases were dyspnea, fever, hemoptysis, chest pain or physical findings with no obvious symptoms. LDH exam showed 4 cases elevation, and $\beta 2$ microglobulin exam showed 2 elevation in these 19 patients.

\section{Chest CT scan}

High resolution computed tomography (HRCT) of the chest (Table 2) revealed unilateral disease in 13 patients
Table 1 Clinical and laboratory features of patients with PPL

\begin{tabular}{lll}
\hline Features & Cases & Percent\% \\
\hline $\begin{array}{ll}\text { Age } \\
<=60\end{array}$ & 13 & 68.4 \\
$>60$ & 6 & 31.6 \\
$\begin{array}{l}\text { Sex } \\
\text { male }\end{array}$ & \\
female & 11 & 57.9 \\
No symptoms & 8 & 42.1 \\
Respiratory symptom & 6 & 31.6 \\
cough, & & \\
dyspnea & 10 & 52.6 \\
fever & 4 & 21.1 \\
hemoptysis & 3 & 15.8 \\
chest pain & 2 & 10.5 \\
$\beta 2$ microglobulin rise & 4 & 21.1 \\
LDH rise & 2 & 10.5 \\
& 4 & 21.1
\end{tabular}

(Fig. 1a-b: showed multiple nodules), bilateral disease in six patients, pneumonia-like (Fig. 1c-d) in five patients, lung single nodule or mass (Fig. 1e) in nine patients and pleural effusion (Fig. 1f: showed multiple nodules with pleural effusion) in four patients. None of these 19 patients accompanied with mediastinal, axillary or cervical lymph node enlargement (Fig. 1a to f).

\section{Bronchoscopy}

Four patients were confirmed by flexible bronchoscopy. Mucosal hyperemia and edema (Fig. 1g) were found in one patient, tumor growth structure (Fig. $1 \mathrm{i}-\mathrm{j}$ ) was found in two patients, tracheal narrow with external pressure (Fig. 1h) was found in one patient. Normal were reported in 15 patients.

\section{Pathologic characteristics}

Pathologic characteristics were performed by pathologist in all 19 cases (Table 3). The lung structure was replaced

Table 2 High resolution computed tomography (HRCT) features

\begin{tabular}{lll}
\hline Features & Cases & Percent\% \\
\hline bilateral disease & 6 & 31.6 \\
unilateral disease & 13 & 68.4 \\
Single nodule or mass & 9 & 47.4 \\
Multiple nodules & 1 & 5.26 \\
Pneumonia-like & 4 & 21.1 \\
Mixed type & 5 & 26.3 \\
$\quad$ Pneumonia-like with cavity & 1 & 5.26 \\
Pneumonia-like with pleural effusion & 1 & 5.26 \\
$\quad$ Nodules with pleural effusion & 3 & 15.8 \\
\hline
\end{tabular}



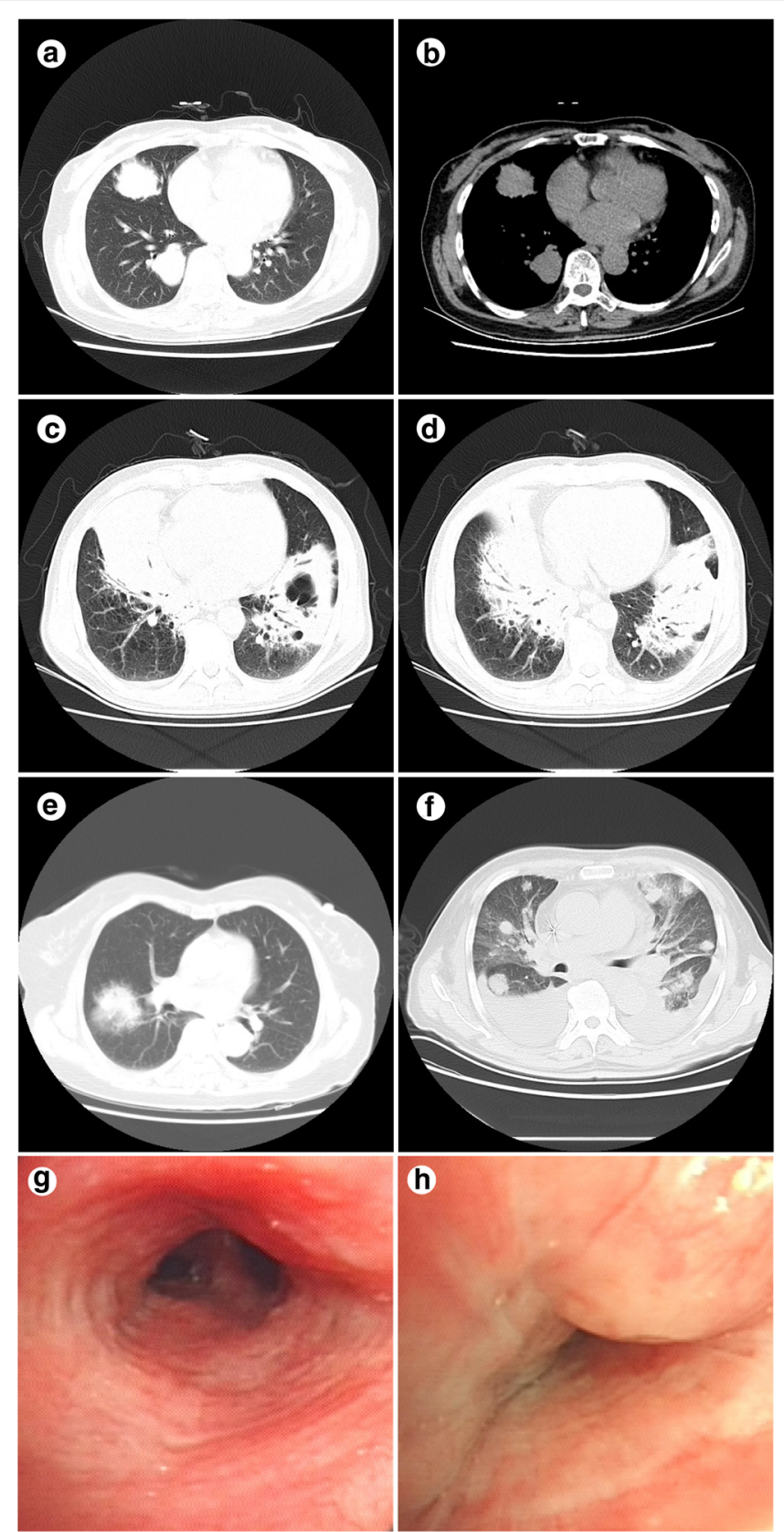

h

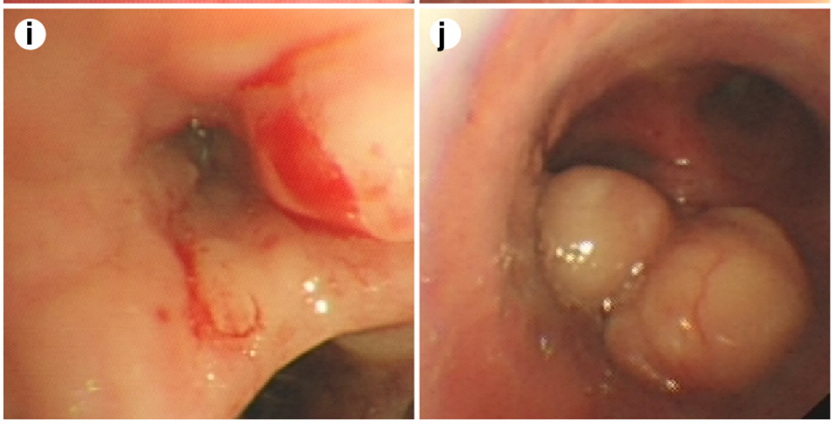

Fig. 1 a-f:chest CT scan (a-b: multiple nodules, c-d:pneumonia-like, e:lung single nodule or mass and pleural effusion, $\mathbf{f}$ : multiple nodules with pleural effusion); $\mathbf{g - j}$ : bronchscopy (g:mucosal hyperemia and edema, $\mathbf{i}-\mathbf{j}$ : tumor growth structure, $\mathbf{h}$ : tracheal narrow with external pressure) 
Table 3 Pathologic types of the 19 cases

\begin{tabular}{lll}
\hline Pathologic type & Cases & Percent\% \\
\hline HL & 2 & 10.5 \\
MALT & 12 & 63.2 \\
Diffuse large B-cell lymphoma & 3 & 15.8 \\
Angioimmunoblastic T-cell lymphoma & 1 & 5.26 \\
ALK (+) anaplastic large cell lymphoma & 1 & 5.26 \\
\hline
\end{tabular}

by tumor cells. Two Hodgkin lymphoma (HL) cases were confirmed to the type of tuberous sclerosis, and 17 non-Hodgkin lymphoma (NHL) cases were confirmed by histopathology. Among $17 \mathrm{NHL}$ cases, 12 cases were confirmed mucosa associated lymphoid tissue B lymphoma type, 3 cases were confirmed diffuse large B- cell lymphoma, one case were confirmed angioimmunoblastic T-cell lymphoma and ALK positive anaplastic large cell lymphoma separately.

\section{Diagnostic procedures and prognosis}

Thirteen patients (68.4\%) were misdiagnosed as pneumonia, lung cancer, and tuberculosis before confirming, and accepted anti-infection, anti- tuberculosis or antitumor treatment. The time from initial diagnosis to the final diagnosis processed from half month up to 2 years, with the medians time of 6 months. Ten patients were receiving antibiotic treatment for presumptive nonresolving pneumonia. Two cases were misdiagnosed as tuberculosis, one has no obvious symptoms but was reported has multiple nodules by CT scan, and accepted anti-tuberculosis treatment for more than 1 year. But during the anti- tuberculosis treatment the lesions processed cavity and then the patient accepted CT-guided percutaneous lung biopsies subsequently, at last PPL was confirmed by histopathology. Another one, whose chief complain was cough, with the imaging of pneumonia-like and T-SPOT positive, accepted antituberculosis treatment for 1 month and ceased voluntarily. So he went back to hospital and accepted biopsy while cough symptoms processed. Another case was misdiagnosed as small cell lung cancer whose bronchoscope found right bronchial neoplasm. Pathology of the biopsy tissue presented poorly differentiated carcinoma, combined with immunohistochemical presented neuroendocrine differentiation, was misdiagnosed as small cell lung cancer treated with EP (etoposide + carboplatin) chemotherapy subsequently. Four monthes later, the patient accepted bronchoscopy biopsy once again for sustained fever, and was confirmed ALK positive anaplastic large cell lymphoma finally (Tables 4 and 5).

Pathological diagnosis was obtained by CT-guided percutaneous lung biopsies in ten patients, while four patients were confirmed by flexible fiberoptic bronchoscopy,
4 cases were confirmed by surgery, and the other one with pleural effusion, were confirmed by biopsies through thoracoscopy.

Prognosis of PPL was generally good, with treatment options ranging from surgical resection in localized cases to chemotherapy in more diffuse involvement. Nineteen patients received various combinations of treatment, surgery plus chemotherapy in four patients, and chemotherapy alone in 15 patients. No patient was treated with radiotherapy. The regimes of chemotherapy were cyclophosphamide, adriamycin, vincristine and prednisone (CHOP), cyclophosphamide, vincristine and prednisone (CVP), rituximab plus CHOP (R-CHOP) and rituximab plus CVP (R-CVP).

\section{Discussion}

PPL $[11,12]$, as an extremely rare disease, represented less than $1 \%$ of lung malignancies, and less than $1 \%$ of non-Hodgkin's lymphoma (NHL) and accounting for only $3.6 \%$ of extranodal lymphomas. There were almost one third of patients (31.6\%) with no symptoms in our research. PPL, with a low incidence, usually occured with nonspecific clinical features, and was highly misdiagnosed in clinic. In our research, 13 patients (68.4\%) were misdiagnosed as pneumonia, lung cancer, tuberculosis before confirming. The present study analyzes the clinical features, laboratory and imaging data, pathologic characteristics, and summarize misdiagnosis reasons of PPL cases. Aims to provide a better understanding and increase the accuracy of early diagnosis and minimize the misdiagnosis of PPL.

The symptoms and physical signs of pulmonary lymphoma were highly heterogeneous. In our research, 11 male and eight female patients were described with a mean age of 51.8 years old (range 19 to 71 years). It occured most commonly around the age of 50-70 years, although rare cases of younger age of onset had been reported. LDH exam showed 4 cases (21\%) elevation, and $\beta 2$ microglobulin exam showed $2(10.5 \%)$ elevation in these 19 patients. Laboratory tests showed no-specific.

Low grade B-cell PPL, as most commonly in PPL, formed almost $85-90 \%$ of all reported cases, out of which almost all cases correspond to MALT-type NHL [13-15]. The recent delineation of new pathologic entities such as low-grade malignant lymphoma of mucosa-associated lymphoid tissue (MALT type) had aided in the understanding of the pathophysiology, clinical course, and management of patients with pulmonary lymphoma. Ahmed [16] reported no clinical symptoms in the MALT lymphoma diagnosis was about 40.9\%. While 63.2\% (12/19) MALT lymphoma were found in our study. The pathology of the six cases with no symptoms were confirmed MALT-type in our study. Nearly half of reported cases of 
Table 4 Misdiagnosed cases

\begin{tabular}{lllll}
\hline Initial diagnosis & Cases & Chief complaint & Time of misdiagnosis(months) & Pathological diagnosis obtained \\
\hline pneumonia & 10 & None, cough, dyspnea or chest pain & $0.5-24$ & $\begin{array}{l}\text { CT-guided percutaneous lung biopsy, } \\
\text { bronchoscopy, surgery and thoracoscopy }\end{array}$ \\
tuberculosis & 2 & Cough, none & $1-12$ & CT-guided percutaneous lung biopsy \\
lung cancer & 1 & Cough and fever & 4 & bronchoscopy \\
\hline
\end{tabular}

MALT-type had been asymptomatic at presentation, while in the others, the presentation had been associated with non specific respiratory symptoms.

The radiographic presentation of PPL was not specific, but variable. The same as Bae et al.'s reported [17], single or multiple nodules or areas of consolidation were the main patterns of radiographic abnormalities in our study, accounted for $47.4 \%$. We found that $26.3 \%$ of patients presented pneumonia-like, with air space consolidation with or without air bronchogram. Other [16-18] radiographic presentation of PPL were pleural effusion, mixed-type, multiple nodules etc. In previous case reports, PPL may appear as a single nodule, as multiple nodules or as cavitated lesions [6]. Cavitated lesions had a wide differential diagnosis including granulomatous diseases (Wegener's granulomatosis), tuberculosis, necrotic pneumonia, pulmonary Gram-negative organisms, anaerobic bacteria or fungal infections, pulmonary abscess, evacuated hydatid cysts, septic emboli, inhalational diseases (eg, coal workers' pneumoconiosis and silicosis), eosinophilic pneumonia, cavitated rheumatoid nodules and neoplastic diseases (primary bronchial carcinoma). Metastatic lung nodules may especially cavitate squamous cell types but also adenocarcinomas, sarcomas, melanomas and osteosarcomas [7]. Because of the wide differential diagnosis, multiple invasive and noninvasive diagnostic investigations were common. Due to these circumstances, in many cases, the invasive biopsy that confirmed the diagnosis was delayed [8].

As the clinical features of PPL were poorly defined, most of the patients were initially misdiagnosed as pneumonia, pulmonary tuberculosis, organizing pneumonia and lung cancer. Altough imaging manifestation of PPL had a less specificity [19-22], there were still some hints: 1) Fuzzy shadow at the edge of lung mass with air bronchogram; 2) Lung mass shadow stable for a long time; 3) Pneumonia-like changing without infections clinical and lab manifestation.

Although there had been several retrospective case reports, few studies were investigated in the Asian population. PPLwas commonly misdiagnosed as pneumonia, pulmonary abscess and carcinoma as reported [9, 10, 23-25].

Table 5 Treatment of 13 misdiagnosed cases

\begin{tabular}{|c|c|c|c|c|}
\hline Case No. & Initial diagnosis & Chief complaint & CT scan & Treatment \\
\hline 1 & Pneumonia & None & Pneumonia-like & anti-infection treatment with levofloxacin for half a month \\
\hline 2 & Pneumonia & None & Nodules with pleural effusion & $\begin{array}{l}\text { anti-infection treatment with cefperazone-sulbactam for } \\
\text { half a month }\end{array}$ \\
\hline 3 & Pneumonia & Cough & Mass & $\begin{array}{l}\text { anti-infection treatment with cefperazone-sulbactam for } \\
\text { a month }\end{array}$ \\
\hline 4 & Pneumonia & Chest pain & Pneumonia-like & $\begin{array}{l}\text { anti-infection treatment with ceftriaxone sodium and } \\
\text { levofloxacin irregularly in local hospital, } 8 \text { months later } \\
\text { he came to our hospital and accepted biopsy }\end{array}$ \\
\hline 5 & Pneumonia & Cough & Pneumonia-like & $\begin{array}{l}\text { anti-infection treatment with ceftriaxone sodium for half a } \\
\text { month, } 6 \text { months later he came back to hospital }\end{array}$ \\
\hline 6 & Pneumonia & Chest pain, dyspea and fever & Pleural effusion & $\begin{array}{l}\text { anti-infection treatment with ceftriaxone sodium for half } \\
\text { a month }\end{array}$ \\
\hline 7 & Pneumonia & None & Mass & anti-infection treatment with levofloxacin for half a month \\
\hline 8 & Pneumonia & Cough, dyspea and fever & Mass & $\begin{array}{l}\text { anti-infection treatment with cefperazone-sulbactam } \\
\text { for half a month }\end{array}$ \\
\hline 9 & Pneumonia & Cough & Mass & anti-infection treatment with levofloxacin for a month \\
\hline 10 & Pneumonia & Cough and chest pain & Mass & $\begin{array}{l}\text { anti-infection treatment with ceftriaxone sodium for half } \\
\text { a month, } 3 \text { months later he came back to hospital }\end{array}$ \\
\hline 11 & Tuberculosis & Cough & Pneumonia-like & $\begin{array}{l}\text { anti-tuberculosis treatment for } 1 \text { month and ceased } \\
\text { voluntarily }\end{array}$ \\
\hline 12 & Tuberculosis & None & Multiple nodules & anti-tuberculosis treatment for more than 1 year \\
\hline 13 & Lung cancer & Cough and fever & Multiple nodules & treated with EP (etoposide + carboplatin) chemotherapy \\
\hline
\end{tabular}


The main cause of misdiagnosis were summarized as follow: 1) The limitation of specialists. All the patients went to the repiratory department because of symptoms or physical findings in lung or air way, some of them accepted antiinfection treatment primarily combined with symptoms, laboratory examination and CT scan. 2) Poor compliance. Some patients didn't come back for subsequent visit when they felt a little better after anti-infection treatment, and some patients went to other hospitals while the symptoms progressed. 3) Some patients' chest CT scan showed pneumonia-like or cavity and the report of T-SPOT was positive, but the evidence of infection were negative, given the highly endemic nature of Mycobacterium tuberculosis in China and the fact of chest CT scan, they accepted diagnostic anti-tuberculosis treatment. 4) The similarity of pathological manifestation. In some cases the pathologists were confused because similarity of pathological manifestation of PPL. In our experience, if a patient was elderly and not responsive to treatment, alternative diagnosis should be considered and invasive procedures, such as bronchoscopy, CT-guided percutaneous lung biopsies, and an open thoracotomy or a video-assisted thoracoscopic (VATS) lung biopsy should be performed.

As a consequence, PPL could be diagnosed only by pathologic methods. Previously, the pathological tissue was mainly obtained through the diseased lung surgery, open thoracotomy, under thoracoscopy biopsy. CT guided percutaneous lung biopsy, thoracotomy and flexible fiberoptic bronchoscopy were the common methods in current. In our stury, pathological diagnosis was obtained by CT-guided percutaneous lung biopsies in ten patients, while four patients were confirmed by flexible fiberoptic bronchoscopy, 4 cases were confirmed by surgery, and the other one clinical feature was pleural effusion, confirmed by thoracoscopy. Of the 19 cases, more than half of the patients' pathology was confirmed by CT guided percutaneous lung biopsy. CT guided percutaneous lung biopsy should be taken actively if the imaging findings did not match the symptoms or the antiinfection treatments to "lung infection" didn't work. Accurate diagnosis requires adequate tissue sampling with appropriate ancillary pathologic studies.

The therapy of PPL relies on histology [26, 27]. Surgery, chemotherapy and radiotherapy were the main treatment to PPL, alone or in combination, as well as therapeutic abstention or follow-up alone, had been commonly used. For MALT lymphoma the final results of IELSG-19 have recently been reported suggesting that rituximab+ chlorambucil should probably be the first line of chemotherapy.

\section{Conclusion}

Clinical and imaging manifestation of PPL is untypical, the symptoms of PPL are mild, systemic symptoms are rare, and about one third of patients may have no symptoms. Imaging manifestation of PPL have a less specificity, but there are still some hints. Biopsies should be taken actively if the imaging findings did not match the symptoms or the anti-infection treatments to "lung infection" didn't work. Accurate diagnosis requires adequate tissue sampling with appropriate ancillary pathologic studies. If clinical manifestation and the diagnosis didn't match, repeated biopsy should be ordered.

\section{Abbreviations \\ CHOP: Cyclophosphamide, adriamycin, vincristine and prednisone; CVP: Cyclophosphamide, vincristine and prednisone; EP: Etoposide + carboplatin; HL: Hodgkin lymphoma; HRCT: Computed tomography; MALT: Mucosa-associated lymphoid tissue; NHL: Non-Hodgkin lymphoma; PPL: Primary pulmonary lymphoma; R-CVP: Rituximab plus CHOP (R-CHOP) and rituximab plus CVP}

\section{Acknowledgements}

Thanks to all the authors and those who have helped in the preparation of the study, and thanks to Zhaona Xu for revising figures and tables.

Funding

No Funding for the study.

Availability of data and materials

All the data and material were true and availability.

\section{Authors' contributions}

All the data and information was summarized and the article was written by DY. The data of chest CT scan was acquired by LZ. The data of Clinical manifestation and laboratory examination Bronchoscopy was collected by PLW and XLG. The data of Pathologic characteristics and Diagnostic procedures and Prognosis was acquired by YFC and LXW. All the data and information was analysed and the conclusion of the article was written by XYH. All authors read and approved the final manuscript.

Ethics approval and consent to participate

Ethical approval for this investigation was obtained from the Research Ethics Committee, the First Affiliated Hospital of Wenzhou Medical University.

\section{Consent for publication}

The study was consent for publication and written by the Ethics committee of the first affiliated hostpital of Wenzhou Medical University. And all patients consent to participate and all data were consent for publish.

Competing interests

The authors declare that they have no competing interests.

\section{Publisher's Note}

Springer Nature remains neutral with regard to jurisdictional claims in published maps and institutional affiliations.

\section{Author details}

${ }^{1}$ The First Affiliated Hospital of Wenzhou Medical University \& Key Laboratory of Heart and Lung, Zhejiang 325035, China. ${ }^{2}$ Wenzhou Medical University,

Zhejiang 325035, China.

Received: 7 August 2017 Accepted: 6 March 2018

Published online: 12 March 2018

References

1. Borie R, Wislez M, Thabut G, et al. Clinical characteristics and prognostic factors of pulmonary MALT Iymphoma. Eur Respir J. 2009:34:1408-16.

2. Nahorecki A, Chabowski M, Straszak E. Et a1. Primary pulmonary MALT lymphoma - case report and literature overview. Eur Rev Med Pharmacol Sci. 2016;20(10):2065-9. 
3. Jóna Á, Illés Á, Szemes $K$, et al. Pulmonary alterations in Hodgkin lymphoma. Orv Hetil. 2016;157(5):163-73.

4. Ankita G, Shashi D. Pulmonary Lymphomatoid granulomatosis- a case report with review of literature. Indian J Surg Oncol. 2016;7(4):484-7. Epub 2016 Apr 29

5. Tanveer S, El Damati A, El Baz A, et al. Primary pulmonary Hodgkin lymphoma. Rare Tumors. 2015;7(4):5968.

6. Diederich S, Link TM, Zühlsdorf H, Steinmeyer E, Wormanns D, Heindel W. Pulmonary manifestations of Hodgkin's disease: radiographic and CT findings. Eur Radiol. 2001;11:2295-305.

7. Lachanas E, Tomos P, Fotinou M, Kalokerinou K. An unusual pulmonary cavitating lesion. Respiration. 2005;72:657-9.

8. Bakan ND, Camsari G, Gur A, et al. A 21-year-old male with productive cough, hemoptysis, chest pain, and weight loss. Respiration. 2007;74:706-9.

9. Ding X, Makino T, Koezuka S, et al. Primary extranodal marginal zone lymphoma of mucosa-associated lymphoid tissue with multiplepure ground-glass opacities:a case report. J Cardiothorac Surg. 2017;12(1):2.

10. Fei $W$, Xiaohong $W$, Hong $Z$, et al. Pulmonary extranodal natural killer T-cell lymphoma (nasal type): a case report and radiological image review. Medicine (Baltimore). 2015;94(38):e1527.

11. Cadranel J, Wislez M, Antoine M. Primary pulmonary lymphoma. Eur Respir J. 2002;20:750-62.

12. Brian B, Graham MD, Douglas J. Primary pulmonary lymphoma. Ann Thorac Surg. 2005;80:1248-53.

13. Tanriverdi E, Acat M, Ozgul G, et al. Primary pulmonary lymphoma: four different and unusual radiologic and clinical manifestations. Leuk Lymphoma. 2017;58(5):1231-3. Epub 2016 Sep 23

14. Chien CC, Lee HS, Lin MH, et al. Primary extranodal natural killer/T-cell lymphoma of bronchus and lung: a case report and review of literature. Thorac Cancer. 2016;7(1):140-4.

15. Pagani M, Antico A, et al. Primary pulmonary high grade non- Hodgkin's lymphoma in an elderly patient. A case report. Tumori. 2007;93:622-4.

16. Ahmed S, Kussick SJ, Siddiqui AK, et al. Bronchial-associated lymphoid tissue Iymphoma: a clinical study of a rare disease. Eur J Cancer. 2004;40(9):1320-6.

17. Bae YA, Lee KS, Han J, Ko YH, Kim BT, Chung MJ, et al. Marginal zone B-cell lymphoma of bronchus-associated lymphoid tissue: imaging findings in 21 patients. Chest. 2008:133:433-40.

18. Li H, Wang T, Wei $X$, et al. Marginal zone B-cell lymphoma of the pulmonary mucosa-associated lymphoid tissue: a case report. Oncol Lett. 2015;10(3):1731-4.

19. Lee J, Park H, Kim YW, et al. Pulmonary lymphoma misdiagnosed as pneumonia. Indian J Hematol Blood Transfus. 2016;32(4):509-11. Epub 2016 Jun 29

20. Lee $\mathrm{S}$, Shin $\mathrm{B}$, Yoon $\mathrm{H}$, et al. A case of primary pulmonary NK/T cell lymphoma presenting as pneumonia. Respir Med Case Rep. 2015;17:1-4.

21. Sirajuddin A, Raparia K, Lewis VA, et al. Primary pulmonary lymphoid lesions: radiologic and pathologic findings. Radiographics. 2016;36(1):53-70.

22. Agarwal KK, Dhanapathi $\mathrm{H}$, Nazar AH, et al. Primary pulmonary lymphomarole of fluoro-deoxy-glucose positron emission tomography-computed tomography in the initial staging and evaluating response to treatment case reports and review of literature. Indian J Nucl Med. 2016:31(3):194-7.

23. Wei S, Li X, Qiu X, et al. Primary lung lymphoma involving the superior vena cava. World J Surg Oncol. 2012;10:131

24. Lluch-Garcia R, Briones-Gomez A, Castellano EM, et al. Primary pulmonary Hodgkin's lymphoma. Can Respir J. 2010;17(6):e106-8.

25. Verma VJ, Jain S, Singhal S, Nallagatla S, et al. Primary pulmonary mucosaassociated lymphoid tissue lymphoma in a patient with acquiredimmune deficiency syndrome. Respir Care. 2011:56(7):1046-9. Epub 2011 Feb 21

26. Zompi S, Coudere L, Cadranel L, et al. Clonality analysis of alveolar B lymphocytes contributes to the diagnostic strategy in clinical suspicion of pulmonary lymphoma. Blood. 2004;103:3208-15.

27. Czuczman MS, Weaver R, Alkuzweny B, et al. Prolonged clinical and molecular remission in patients with low-grade or follicular non-Hodgkin's lymphoma treated with rituximab plus CHOP chemotherapy: 9-year followup. J Clin Oncol. 2004;22:4711-6.

\section{Submit your next manuscript to BioMed Central and we will help you at every step:}

- We accept pre-submission inquiries

- Our selector tool helps you to find the most relevant journal

- We provide round the clock customer support

- Convenient online submission

- Thorough peer review

- Inclusion in PubMed and all major indexing services

- Maximum visibility for your research

Submit your manuscript at www.biomedcentral.com/submit
C) Biomed Central 